\title{
TOXOPLASMOSIS ENTRE MUJERES EMBARAZADAS EN CIUDAD DE LA HABANA
}

\author{
R. López ${ }^{1}$, Xiomara Pérez $^{2}$, E. Guerra ${ }^{1}$, R. Herrera ${ }^{2}$ y Carmen Acosta ${ }^{2}$
}

\begin{abstract}
Se realizó un estudio seroepidemiológico sobre 3196 gestantes, residentes en Ciudad de La Habana, con la finalidad de conocer la presencia de anticuerpos IgG anti-Toxoplasma gondii; sus concentraciones, expresadas en $u \mathrm{i} / \mathrm{mL}$, y el número esperado de infección primaria. El $50,59 \%$ de las gestantes resultaron positivas a T. gondii. El $37,41 \%$, del total de las encontradas positivas, presentan concentraciones de anticuerpos específicos superiores a $100 \mathrm{ui} / \mathrm{mL}$. Se sugiere la valoración de aspectos fundamentales relacionados con esta infección parasitaria dentro del Programa Nacional de Atención a las Embarazadas.
\end{abstract}

Palabras claves: toxoplasmosis; gestantes; Ciudad de La Habana.

\section{INTRODUCCION}

A partir del primer informe sobre toxoplasmosis en nuestro país (1), diferentes autores se propusieron conocer la real magnitud de esta infección en algunos grupos de personas mediante la detección de anti-Toxoplasma gondii.

La epidemiología de esta infección parasitaria todavía requiere de análisis, así como de un esfuerzo en la red de salud nacional para consolidar el diagnóstico en aquellas áreas asistenciales que lo tienen y extenderlo hacia otras. Conjuntamente con ello debemos buscar la centralización de los datos para su procesamiento estadístico, pues sólo así podemos precisar si un programa nacional de prevención y control dela toxoplasmosis se justifica.

En este trabajo consideramos de especial interés el empleo de un método de estimación teórica, en un estudio seroepidemiológico, sobre la relación toxoplasmosis-gravidez en ciudad de La Habana, Cuba.

\section{MATERIALES Y METODOS}

Se colectaron 3.196 sueros de una población de mujeres sanas, comprendidas desde los 15 hasta los 44 años de edad y con 15 a 19 semanas de embarazo. Todas ellas residen en ciudad de La Habana y acudieron al Hospital Gineco-obstétrico Ramón Gonzalez Coro a un chequeo médico sistemático en el marco del programa nacional de prevención y detección de malformaciones congénitas en las gestantes.

Los sueros fueron procesados en busca de anticuerpos Ig G anti-Toxoplasmagondiimediante la técnica del UMELISA. (2).

Para el procesamiento de los datos, agrupados en intervalos de cinco años, se empleó el método de comparación múltiple de proporciones y en el caso en que existía diferencia significativa, la dócima de Duncan $(P<0,05)$ para conocer entre qué niveles del parámetro en estudio se manifestó esa diferencia.

1 Centro Nacional de Biopreparados. Aptdo 6048. Habana 6.Cuba

2 Centro de Inmunoensayo. Ave 25 y 154. Aptdo 6945. Habana 6 
Se empleó un modelo teórico, (3), para determinar el número esperado de infección primaria sobre la población de gestantes en Ciudad de La Habana. Los datos sobre la población cubana, pertenecientes al año 1991, fueron obtenidos del Instituto de Investigaciones Estadísticas. (4).

Este modelo se basa en la hipótesis de que cada individuo, de una población dada, está sujeto a un riesgo $r$, que es la probabilidad de estar en contacto con el Toxoplasma gondii en el transcurso de un año. De esta manera, corresponde con la tasa de seroconversión, pues ésta se produce al primer contacto.

Las probabilidades $P x$, de que un individuo sea seropositivo a la edad $x, y, \mathrm{x}$, de que un individuo sea seronegativo a la edad $x$, se definen como.

$$
\begin{aligned}
& P_{x}=\sum_{i-1}^{x} r(1-r)^{i-1} \\
& Q_{x}=(1-r)^{x}
\end{aligned}
$$

A partir de estas ecuaciones se obtuvieron los valores de las estimaciones puntuales de los riesgos por edades $r_{\times}$y sus respectivas varianzas $\mathrm{s}_{\mathrm{x}}$. Para una mejor estimación del riesgo de seroconversión $r$, se utilizó el método de máxima verosimilitud. El cálculo del máximo de la derivada de la función de máxima verosimilitud con respecto a $r$, se obtuvo mediante el método de la búsqueda dicotómica. Además, se analizó la distribución de las desviaciones de los riesgos por edades con respecto a la estimación de máxima verosimilitud de $r$ mediante la expresión

$$
\left(r_{x}-r\right) / s^{2} \text {. }
$$

Para realizar la comparación de las frecuencias observadas y esperadas de seronegatividad por edad, se utilizaron la prueba $x^{2}$ de bondad de ajuste y la prueba de Kolmogorov-Smirnov.

Una vez obtenidos los valores de r y de $Q_{x}$, se calcularon los valores esperados de seroconversiones maternas por edades mediante la expresión

$$
V E S_{x}=r Q_{x} G_{x}
$$

donde $G_{x}$ es la cantidad de mujeres gestantes por edades, en Ciudad de La Habana en 1991.
Por último se obtuvieron los porcentajes de seroconversión materna por edades, como el porciento que representa $V E S_{x}$ del total $G_{x}$.

\section{RESULTADOS Y DISCUSION}

La tabla 1 nos pone de manifiesto que de los 3.196 sueros evaluados, el $50,59 \%$ resultaron positivos a la presencia de anticuerpos IgG antiToxoplasmagondii. Anteriormente, Blanco y colab (5), empleando la técnica de microELISA hallaron un $62,10 \%$ de positividad a $T$. gondii. en sueros de madres en el momento del parto. Esta diferencia bien pudiera estar influenciada por el número de muestras analizadas en cada caso (196 vs 3.196) o tal como ha sido indicado por Foulon et al, (6), la causa pudiera ser: el cambio en la fuerza de la infección por el tiempo.

Tabla 1. Frecuencias observadas por edades

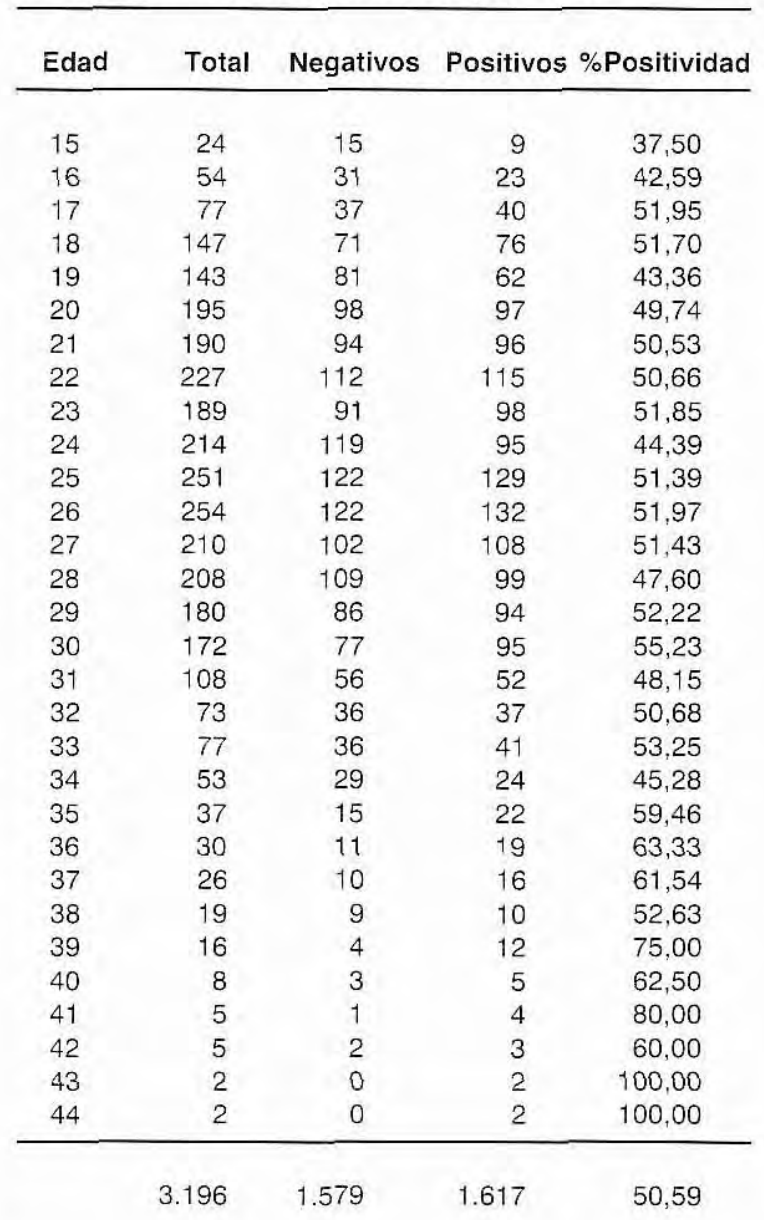


También puede observarse que desde los 16 años de edad la incidencia de anticuerpos específicos entre las gestantes es superior al $40 \%$, siendo esto indicativo de que la infestación por esta parasitosis, en un apreciable porcentaje, se realiza desde edades tempranas en las mujeres que viven en la capital del país. (7)

Del total de las gestantes, el $84,11 \%(2.688 /$ 3.196) se insertan entre los 18-31 años de edad; hecho que indica que la mayoría de los nacimientos se cumplen en madres jóvenes con plenitud de desarrollo de sus órganos sexuales, pero al mismo tiempo ese gran número de mujeres manifiestan una negatividad en el orden del $46,50 \%$ (1.250/2.688); enseñándonos que una cifra considerable de mujeres gestantes en su segundo trimestre de embarazo, son susceptibles de contraer una infección primaria a $T$. gondii.

La población fue además analizada por grupos, con 5 años de intervalos entre ellos. (Tabla 2). Esto nos mostró que la tasa de seropositividad/ edad tiende al aumento, desde $47,19 \%$ hasta $72,73 \%$.

Al someter estos valores a un análisis estadístico ello nos hace conocedores de que la presencia de anticuerpos IgG a $T$. gondii en los individuos considerados en las edades de 15-19 años (210/445); 20-24 años (501/1.015); 25-29 años (562/1103) y 30-34 años (249/483), no difieren entre sí $(P>0,05)$. Así mismo, éstos difieren significativamente $(P<0,05)$ con las eda- des de 35-39 años (79/128) y de 40-44 años (16/ 22), las cuales también difieren entre sí.

Al aumentar la edad, el tiempo probable de exposición a T.gondii también aumenta y con ello se dan muchas más oportunidades para que los sujetos negativos puedan infectarse.

Para el intervalo de 25-29 años de edad, encontramos un valor medio de positividad semejante al alcanzado por la totalidad de las gestantes examinadas.

Al analizar las concentraciones de anticuerpos específicos, expresados en $\mathrm{ui} / \mathrm{mL}$, entre las gestantes consideradas positivas, vemos que el $62,59 \%$ de las mismas se insertan desde una infección sobrepasada con su simple secuela serológica hasta la de una posible fase incipiente. Generalmente en estas mujeres la infección por $T$. gondii ha tenido lugar meses o incluso años antes de la toma de la muestra de la sangre y no se esperan cambios en sus tasas de anticuerpos anti-Toxoplasma gondii.

El $37,41 \%$ presenta anticuerpos específicos entre 100->200 ui/mL, pudiendo interpretarse ello como concentraciones de valores medios, siendo representantes de una fase de probable actividad. Esta situación es de consideración, pues el número de gestantes enmarcada en esta fase, (605/1.617), representa un poco más de la tercera parte de las encontradas positivas a $T$. gondii y el $18,92 \%$, (605/3196), del total de ellas.

Tabla 2. Presencia de anticuerpos lgG anti-T. gondii en relación a diferentes rangos de edades.

\begin{tabular}{|c|c|c|c|c|c|c|c|c|}
\hline \multirow[b]{2}{*}{$\begin{array}{l}\text { Rango edad } \\
\text { (años) }\end{array}$} & \multirow[b]{2}{*}{$\begin{array}{c}\text { Número } \\
\text { indiv. }(\%)\end{array}$} & \multirow[b]{2}{*}{$\begin{array}{l}\text { Número } \\
\text { negat.(\%) }\end{array}$} & \multicolumn{6}{|c|}{ u. $\mathrm{i} / \mathrm{mL}$ de anticuerpos IgG anti-T. gondii. No. indiv. y (\%) } \\
\hline & & & $\begin{array}{l}>12,5 \\
<25,0\end{array}$ & $\begin{array}{l}\geq 25,0 \\
<50.0\end{array}$ & $\begin{array}{l}\geq 50,0 \\
<100\end{array}$ & $\begin{array}{l}\geq 100 \\
<200\end{array}$ & $\geq 200$ & $\begin{array}{l}\text { Número } \\
\text { posit.(\%) }\end{array}$ \\
\hline $15-19$ & $445(13,92)$ & $235(52,81)$ & 21 & 36 & 57 & 68 & 28 & $210(47,19)^{\mathrm{c}}$ \\
\hline $20-24$ & $1.015(31,76)$ & $514(50,64)$ & 51 & 94 & 157 & 126 & 63 & $501(49,36)^{c}$ \\
\hline $25-29$ & $1.103(34,51)$ & $541(49,05)$ & 44 & 122 & 199 & 137 & 60 & $562(50,95)^{\circ}$ \\
\hline $30-34$ & $483(15,11)$ & $234(48,45)$ & 22 & 65 & 85 & 51 & 26 & $249(51,55)^{c}$ \\
\hline $35-39$ & $128(4,01)$ & $48(38,28)$ & 9 & 15 & 25 & 21 & 9 & $79(61,72)^{\mathrm{b}}$ \\
\hline $40-44$ & $22(0,69)$ & $6(27,27)$ & 1 & 6 & 3 & 5 & 1 & $16(72,73)^{\mathrm{a}}$ \\
\hline & 196 & $1.579(49,41)$ & $\begin{array}{c}148 \\
(9,15)\end{array}$ & $\begin{array}{c}338 \\
(20,90)\end{array}$ & $\begin{array}{l}526 \\
(32,53)\end{array}$ & $\begin{array}{c}418 \\
(25,85)\end{array}$ & $\begin{array}{l}187 \\
(11,56)\end{array}$ & $1.617(50,59)$ \\
\hline
\end{tabular}


En todos los casos seropositivos se hace necesario determinar la presencia de IgM específica y/o repetir la evaluación de la IgG específica 3-4 semanas más tarde para establecer si existe diferencia, en sentido positivo, entre las concentraciones de estos sueros pareados. De suceder esto último, ello indicaría que nos encontramos en la rama ascendente de la curva de expresión de la evolución de los anticuerpos específicos, lo que sería igual a una primo infección. El hecho de encontrar IgM no es absolutamente indicativo de encontrarnos ante un cuadro agudo de la enfermedad, pues Naot et al, (8), han indicado que estos anticuerpos persisten en la sangre por espacio de 3-9 meses después de la infección y en algunas ocasiones por tiempo mayor.

Tan pronto se tengan elementos que indiquen que la mujer gestante ha sido infectada durante el embarazo actual, debe administrarse, esencialmente, Spiramicina. El tratamiento reduce la transmisión al feto pero no reduce la severidad de la infección transmitida. (9).

Para determinar el número esperado de infección primaria sobre la población de gestantes estudiadas, vemos que: los valores de la estimación puntual de $r_{x}$ varían entre 0,0176 y 0,0420 , siendo el promedio de estas estimaciones puntuales de 0,0281. (Tabla 3). En esta tabla también se muestran los valores observados y esperados de negatividad, así como las varianzas de los riesgos por edades.

La estimación de máxima verosimilitud de $r$, dió como resultado $r=0,0277$, con un intervalo de confianza del $95 \%$ igual a 0,0256-0,0306. Al estudiar la variabilidad de los riesgos por edades con respecto a $r$, se encontró que $\left(r_{x}-r\right) / s^{2}$, sigue una distribución, aproximadamente, normal con media 1,08 y varianza 0,8 . Además no se encontró ningún valor que con respecto a la media tuviera una desviación mayor que dos veces la desviación estándar.

Al realizar la comparación entre las frecuencias observadas y esperadas de negatividad, mediante el uso de la prueba de bondad de ajuste, se obtuvo un valor de $x^{2}=26,22$, que demuestra que no hay diferencias significativas $(P>0,05)$ entre estas frecuencias. Este resultado se reafirma mediante al uso de la prueba de Kolmogorov-Smirnov, pues se obtuvo un valor de $D=0,0465$, que tampoco es significativo. Ello nos permite considerar que el modelo es aceptable para la determinación de los riesgos de seroconversión en nuestras gestantes.

Tabla 3. Riesgos de seroconversión por edades (estimaciones puntuales). Valores esperados y observados de negatividad calculados a partir de la estimación de máxima verosimilitud de r)

\begin{tabular}{|c|c|c|c|c|c|}
\hline Edac & $\begin{array}{l}\text { d Riesgos } \\
\text { por edades }\end{array}$ & $\begin{array}{l}\text { Varianza } \\
s \quad(10-5)\end{array}$ & $\begin{array}{l}\text { a Negativos } \\
\text { Observados }\end{array}$ & $\begin{array}{c}\text { Negatividad } \\
\%\end{array}$ & $\begin{array}{l}\text { Negativos } \\
\text { Esperados }\end{array}$ \\
\hline 15 & 0,0308 & 10,4 & 15 & 62,5 & 15,7 \\
\hline 16 & 0,0341 & 5,0 & 31 & 57,4 & 34,4 \\
\hline 17 & 0,0422 & 4,5 & 37 & 48,1 & 47,7 \\
\hline 18 & 0,0396 & 2,1 & 71 & 48,3 & 88,6 \\
\hline 19 & 0,0295 & 1,4 & 81 & 56,6 & 83,8 \\
\hline 20 & 0,0338 & 1,2 & 98 & 50,3 & 111,1 \\
\hline 21 & 0,0330 & 1,1 & 94 & 49,5 & 105,3 \\
\hline 22 & 0,0316 & 0,9 & 112 & 49,3 & 122,3 \\
\hline 23 & 0,0313 & 1,0 & 91 & 48,1 & 99,0 \\
\hline 24 & 0,0242 & 0,6 & 119 & 55,6 & 109,0 \\
\hline 25 & 0,0284 & 0,6 & 122 & 48,6 & 124,3 \\
\hline 26 & 0,0278 & 0,6 & 122 & 48,0 & 122,3 \\
\hline 27 & 0,0264 & 0,7 & 102 & 48,6 & 98,3 \\
\hline 28 & 0,0228 & 0,5 & 109 & 52,4 & 94,6 \\
\hline 29 & 0,0251 & 0,7 & 86 & 47,8 & 79,6 \\
\hline 30 & 0,0264 & 0,8 & 77 & 44,8 & 74,0 \\
\hline 31 & 0,0210 & 0,9 & 56 & 51,9 & 45,2 \\
\hline 32 & 0,0218 & 1,3 & 36 & 49,3 & 29,7 \\
\hline 33 & 0,0228 & 1,3 & 36 & 46,8 & 30,4 \\
\hline 34 & 0,0176 & 1,3 & 29 & 54,7 & 20,4 \\
\hline 35 & 0,0255 & 3,1 & 5 & 40,5 & 13,8 \\
\hline 36 & 0,0275 & 4,2 & 11 & 36,7 & 10,9 \\
\hline 37 & 0,0255 & 4,3 & 10 & 38,5 & 9,2 \\
\hline 38 & 0,0195 & 3,9 & 9 & 47,4 & 6,5 \\
\hline 39 & 0,0349 & 11,5 & 4 & 25,0 & 5,3 \\
\hline 40 & 0,0242 & 12,4 & 3 & 37,5 & 2,6 \\
\hline 41 & 0,0385 & 44,0 & 1 & 20,0 & 1,6 \\
\hline 42 & 0,0216 & 16,3 & 2 & 40,0 & 1,5 \\
\hline 43 & 0,0000 & 0,0 & 0 & 0,0 & 0,6 \\
\hline 44 & 0,0000 & 0,0 & 0 & 0,0 & 0,6 \\
\hline
\end{tabular}

*Promedio de los riesgos por edades. 
La predicción de los porcentajes de adquirir toxoplasmosis durante el embarazo, fueron obtenidos de la distribución por edades de la población total de mujeres embarazadas (30.936) en Ciudad de La Habana en el año 1991 y de la estimación de máxima verosimilitud de $r$.

Se obtuvo que los porcentajes de adquirir toxoplasmosis durante el embarazo, disminuyen desde $1,82 \%$ para 15 años, hasta $0,80 \%$ para 44 años. Además se encontró, globalmente, un valor esperado de $425(1,37 \%)$ seroconversiones maternas por años. (Tabla 4).

Tabla 4. Distribución por edades de adquirir toxoplasmosis (A.T.) Porciento esperado de A.T durante el embarazo.

\begin{tabular}{|c|c|c|}
\hline Edad & $\begin{array}{c}\text { No esperado } \\
\text { de A.T. }\end{array}$ & $\begin{array}{l}\% \text { esperado de A.T. } \\
\text { durante el embarazo }\end{array}$ \\
\hline 15 & 4,74 & 1,82 \\
\hline 16 & 10,94 & 1,77 \\
\hline 17 & 14,64 & 1,71 \\
\hline 18 & 18,59 & 1,67 \\
\hline 19 & 21,67 & 1,62 \\
\hline 20 & 25,35 & 1,58 \\
\hline 21 & 28,19 & 1,54 \\
\hline 22 & 30,03 & 1,49 \\
\hline 23 & 28,82 & 1,45 \\
\hline 24 & 28,88 & 1,41 \\
\hline 25 & 43,79 & 1,37 \\
\hline 26 & 34,63 & 1,33 \\
\hline 27 & 26,43 & 1,28 \\
\hline 28 & 22,18 & 1,26 \\
\hline 29 & 18,79 & 1,23 \\
\hline 30 & 13,89 & 1,19 \\
\hline 31 & 11,58 & 1,16 \\
\hline 32 & 10,61 & 1,13 \\
\hline 33 & 7,72 & 1,10 \\
\hline 34 & 6,68 & 1,07 \\
\hline 35 & 4,60 & 1,04 \\
\hline 36 & 3,75 & 1,00 \\
\hline 37 & 3,03 & 0,98 \\
\hline 38 & 2,18 & 0,95 \\
\hline 39 & 1,40 & 0,93 \\
\hline 40 & 0,88 & 0,90 \\
\hline 41 & 0,48 & 0,86 \\
\hline 42 & 0,33 & 0,85 \\
\hline 43 & 0,18 & 0,82 \\
\hline 44 & 0,05 & 0,80 \\
\hline
\end{tabular}

Los porcentajes de adquirir toxoplasmosis y los valores esperados de seroconversión por edades (ajustados mediante un polinomio de grado seis) se muestran en la figura 1.

Según lo propuesto por Desmont,(10) y Frenkel, (11), esto representaría aproximadamente 99-148 casos $(3,2-4,8 / 1.000)$ de toxoplasmosis congénita anual entre los niños, sobre la base de una tasa de incidencia del $30-45 \%$. De estos niños infectados, el $15 \%$ (15-22) deben presentar daños clínicos severos y un $19 \%$ adicional (19-28) padecerán una enfermedad ligera.

Tomando en consideración que el gasto económico promedio por enfermedad en los Estados Unidos en el año 1981 fue de aproximadamente \$ 100.000 durantelavida de un niño contoxoplasmosis congénita (11), y que los datos ofrecidos en este trabajo sólo son representativos del daño que puede causar esta antropozoonosis en las gestantes de la

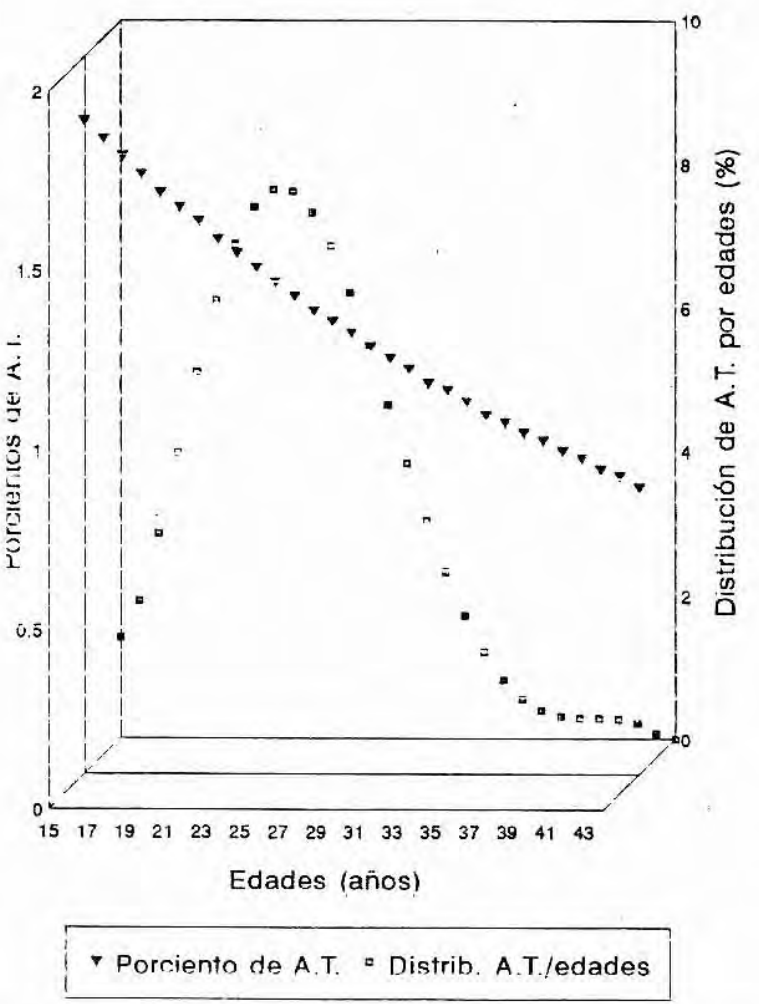

Figura 1. Porcentajes esperados y distribución por edades de adquirir toxoplasmosis (A.T) durante el embarazo. 
capital del país, entonces esta entidad parasitaria bien pudiera ser enmarcada en la categoría de "situación de salud para ser valorada nacionalmente entre las gestantes".

Con vista a reducir los efectos dañinos que puede provocar esta antropozoonosis, nuestro trabajo debe estar dirigido principalmente sobre aquella parte de la población femenina embarazada que evidencie pruebas serológicas negativas a $T$. gondii entre las $8-12$ semanas de gestación, precisándose qué medidas profilácticas deben extremarse para limitar las posibilidades de infección primaria. De ahi, la necesidad de que el diagnóstico de anticuerpos especifícos a $T$. gondii se realice tempranamente durante el período de gestación.

Toda la información a la futura madre debe ofrecérsele de forma clara y sencilla, debiéndose apuntar la importancia de acudir a las sucesivas pruebas serológicas y a las consultas médicas pues ellas son la garantía para detectar una posible infección inicial y recibir indicaciones facultativas (12).

Aquella gestante en que se detectara una seroconversión a $T$. gondii sería impuesta de tratamiento y su hijo debe ser examinado, seguido serológicamente y por evidencias clínicas, al menos durante el primer año de vida, aunque no presentara signos ni síntomas adjudicables a una toxoplasmosis congénita.

También se requiere que aspectos fundamentales como la transmisión, patogenia, respuesta inmune, prevención y tratamiento, deban ser concienzudamente evaluados en el marco del programanacional que da cobertura de atención a todas las embarazadas. Ello daría conocimiento pleno del impacto de la toxoplasmosis en la red perinatal e infantil en nuestro país.

\section{SUMMARY}

A seroepidemiological study including a population of 3,196 pregnant women was carried out in Havana City, to identify the presence of IgG/anti-Toxoplasma gondiiantibodies, their concentration (ui/mL) and the expected number of primary infection. $50.59 \%$ of the pregnant women were positive to $T$. gondii. $37.41 \%$, of the total positives, reported concentrations of specific antibodies greater than $100 \mathrm{ui} / \mathrm{mL}$. The evaluation of fundamental aspects related to this parasite infection is urgently recommended as part of the National Programme for Pregnant Women.

Keywords: toxoplasmosis; pregnant women; Havana.

\section{REFERENCIAS}

1. Campuzano J. Un caso de toxoplasmosis canina en La Habana. Bol Ofic Secret San Benef 1913; $(9,1-6)$ : 620. En-jun. La Habana.

2. Acosta Carmen Pérez X. Herrera R. et al. Evaluación clínica del UMELISA-Toxoplasma. Congreso Biotecnología Habana 1992. Resumen 19,15 junio 8-12.

3. Papoz L, Simondon F, Saurin W, et al. A simple model relevant to toxoplasmosis applied to epidemiologic results in France. Am J Epimediology 1986; 123; 1.

4 Instituto de Investigaciones Estadisticas. Estudios y datos sobre la población cubana. 1991; publicación No 21 (en proceso de edición) Ciudad de La habana. Cuba.

5. Blanco R, Malberty A, López R, et al. Estudio de la presencia de anticuerpos contra Toxoplasma gondii en sueros de madres y recién nacidos en el momento del parto. Congreso 50. Aniversario del Instituto de Medicina Tropical Pedro Kouri.1988. Resumen T319: 137.

6. Foulon W, Naessens A, Lauwers $\mathbf{S}$, et al. Impact of primary prevention on the incidence of toxoplasma during pregnancy. Obst. Gynecol. 1988; 72: 363.

7. López R, Pérez X, Font L, et al. Presencia de anticuerpos anti-T. gondii en la población de Ciudad de La Habana. Rev. Ibérica de parasitología. 1993 en prensa.

8. Naot Y, Guptill D.R, Remington J.S. Duration of IgM antibodies to toxoplasma gondii after acute acquired toxoplasmosis. J Infect Dis 1982; 145:770.

9. Desmont G.Toxoplasmose acquise de la femme enciente. Lyon Medical 1982; 248:115

10. Desmont G, et Couvrier J. Congenital toxoplasmosis: A prospective study of 378 pregnancies. N Engl J Med $1974 ; 290: 1110$.

11. Frenkel J.K, Congenital toxoplasmosis. Prevention palliation. Am J Obst Gyn. 1981;141:359.

12. Blanco R, Malberti J. A, López R, et al. La vigilancia deagentes biológicosenlasembarazadas. Toxoplasmosis. Documento orientador. Dpto. Perinatologia Básica. Instituto Superior de Ciencias Médicas de la Habana. 1989. 